\title{
Il diritto laico come diritto fondato sulla forza e sul potere
}

\author{
Secular Law as a Law Based on Strenght and Power
}

\author{
Gaetano Lo CASTRO \\ Università di Roma «La Sapienza» (Italia) \\ gaetano.locastro@gmail.com
}

\begin{abstract}
Riassunto: Questo contributo, chiarito che non esiste «lo Stato laico» bensì tanti Stati laici quanto sono gli ordinamenti che hanno deciso di separare l'ordine temporale da quello spirituale, secondo le fogge storicamente più diverse e cangianti, va alla radice del problema scaturente dalla tensione - insita nel fenomeno giuridico - tra kratos (potere, forza), ed ethos (giustizia, diritto come limite al potere). In particolare, approfondisce sul piano storico e teoretico la funzione di giudizio del potere (e di conseguenza, riconduzione a ragione della forza, e di moralizzazione della politica) che riveste la religione, e chiarisce l'attuale ambiguità semantica del termine laico, in una cultura giuridico-politica (anche nel diritto canonico) fortemente condizionata dal positivismo imperante e dall'assenza di efficaci limiti al dilagare del potere.
\end{abstract}

Parole chiave: laicità; diritto; giustizia; potere; moderrnità.

\begin{abstract}
This paper, clarified that there is no «secular state» but so many lay states as the ordinances that have decided to separate the temporal order from the spiritual one, according to the historically diverse and changing variables, is at the root of the problem arising from the tension -inherent in the legal phenomenon-; between kratos (power, strength), and ethos (justice, law as a limitation to the power). In particular, he studies in the historical and the theoretical way the function of judgment of power (and, consequently, the reawakening of force and moralization of politics) that governs religion, and clarifies the current semantic ambiguity of the secular term in one political-juridical culture (even in canon law) strongly conditioned by the dominant positivism and the absence of effective limits to the spread of power.
\end{abstract}

Keywords: laicity; law; justice; power; modernity.

O ve si escluda ogni forzatura ideologica, sembrerebbe che nell'epoca moderna alla laicità altro significato rigoroso non possa essere attribuito se non quello di rappresentare la reciproca autonomia delle realtà temporali e delle realtà spirituali. Un'autonomia che poi prende forme diverse, secondo le opzioni storiche delle varie organizzazioni statuali, che saranno manifestate dal loro specifico diritto. Onde non un unico tipo di diritto «laico» potrà aversi, ma tanti diritti laici quanti gli Stati che vivono in un rapporto di alterità rispetto all'ordine spirituale, secondo il rispettivo modo di vivere l'aconfessionalità.

Così, l'aconfessionalità, o se si vuole, la laicità possono indurre - come avviene nella gran parte degli ordinamenti dell'Europa occidentale - a teorizzare e a costruire le relazioni fra ordine temporale e ordine spirituale come relazioni fra gli enti istituzionali (lo Stato, la Chiesa e le altre confessioni), 
che tali ordini rappresentano ed organizzano all'interno della società globale; e conseguentemente possono indurre a trattare i loro rapporti nell'ambito dell'ordinamento internazionale, o comunque come fra soggetti esterni l'uno all'altro - con la conseguente elaborazione di un diritto ispirato a una visione internazionalistica del problema, per la quale il fattore religioso resterebbe estromesso, in linea di principio, dall'ordinamento statale, in questo potendo assumere rilievo se ed in quanto lo stesso ordinamento, in modo libero e sovrano, glielo consenta -.

Tuttavia aconfessionalità e laicità si hanno anche, in forme diverse, là dove - è il caso di molti degli Stati americani confederati - la dimensione religiosa sia in prevalenza considerata e protetta per i suoi profili di libertà individuale, mentre resta assoggettata al diritto comune per gli specifici aspetti istituzionali.

Benché poi possa sembrare paradossale, aconfessionalità e laicità si hanno anche, tutto sta ad intendersi, là dove - come nel Regno Unito d'Inghilterra la distinzione fra gli ordini parrebbe compromessa per avere essi il medesimo capo, la Chiesa essere ritenuta Chiesa di Stato e il Parlamento essere chiamato a deliberare sui suoi fatti interni (e così deliberare, ad esempio, sul problema squisitamente religioso, se le donne possano o non possano essere ammesse al sacerdozio $)^{1}$.

E dunque la laicità del diritto può supporre ora un regime di separazione, ora di indifferenza istituzionale, ora di sopraffazione o comunque di subordinazione dell'ordine spirituale al temporale. L'elemento accomunante, in queste diverse e, per certi aspetti, opposte concezioni, parrebbe essere costituito dalla riduzione del rilievo dell'ordine spirituale nella società globale, in cui esso insiste con l'ordine temporale, o nella sua mortificazione.

Per quanto però condizionati dalla moderna cultura, che ne ha fatto un problema di politica ecclesiastica, di posizione che lo Stato deve assumere nei confronti della Chiesa e del fenomeno religioso, noi tutti avvertiamo che il problema della laicità non può essere racchiuso in categorie storicamente condizionate, quali quelle sopra enunciate; che esso ha qualcosa di universale, che

1 In questo caso qualche autore preferisce parlare di «secolarizzazione», piuttosto che di «laicizzazione»; questa sarebbe fenomeno proprio dei paesi di tradizione cattolica, ove la Chiesa si pone come società totale rispetto allo Stato; quella (secolarizzazione) dei paesi di tradizione protestante, ove le religioni, create in seguito alla Protesta, sono costituite in istituzioni interne allo Stato e con questo si evolvono: cfr. CHAMPION, F., «Entre laïcisation et sécularisation. Des rapports Église-Etat dans l'Europe communautaire», in Le débat, 77 (nov.-déc. 1993), p. 48. 
ne fa un problema di tutte le epoche, presente in modo drammatico nella realtà umana presso tutte le latitudini, ben oltre le dimensioni provinciali entro le quali noi siamo abituati a vederlo e a trattarlo.

Ebbene, la questione essenziale della laicità, quella che ne esprime e ne assicura l'universalità, è disvelata dal rapporto dialettico, che si manifesta in costante tensione, fra il potere, la forza, il mondo del kratos, da un lato, e il mondo dell'ethos, della giustizia, del diritto (in quanto di tale mondo espressioni), dall'altro; fra il potere, la forza, e il loro limite, se un limite al potere ed alla forza può essere assegnato.

Trattasi di un problema ancor più universale rispetto a quello del rapporto fra legge e coscienza, tra dimensione soggettiva e dimensione oggettiva, tra l'io e l'insieme, tra il pubblico e il privato, tra la società civile e la religiosa, tra lo Stato e la Chiesa, tutti costituenti profili dualistici della realtà umana, ma più specifici rispetto al problema qui rappresentato, sebbene da questo (dalle soluzioni a questo date) condizionati in profondità.

Per quanto il problema del rapporto fra il potere e il suo limite sembrerebbe oggi non occupare la ribalta dei dibattiti dottrinali, e per quanto i giuristi, di certo fra i più interessati alla sua corretta trattazione e alle sue soluzioni, si siano dati assenti, o siano rimasti in una posizione subalterna rispetto ad altri maîtres à penser, pure è indubitabile che nell'anzidetto problema sono come racchiusi tutti i grandi temi della civile convivenza.

Che rapporto v'è tra il potere, che ha la forza (e che per taluni è la forza), e il suo limite (che, soltanto in quanto limite della forza, noi potremo davvero denominare etica, giustizia, diritto)? Che rapporto si dà tra il kratos e l'ethos, nel cui contrasto s'identifica, come è stato detto ${ }^{2}$, la storia dell'Occidente?

Per secoli il mondo occidentale è stato abituato a vedere nella Chiesa, o, se si preferisce, nel messaggio cristiano, un limite oggettivo al potere; si è arrivati addirittura ad identificare con i contenuti di tale messaggio la dimensione dell'eticità, ed in essa s'è vista nascere l'idea del diritto come drictum agere.

Il rigetto di tale limite, rappresentato dalla Chiesa, come di ogni altro pensabile limite, fuori della Chiesa, rientra nella dinamica naturale del potere, ne rappresenta, si può dire, una sua connaturale tensione. Tale rigetto, che può avvenire in forme occulte e non organizzate, ma può aversi con formali espressioni ed essere financo teorizzato in costruzioni razionali, svela il de-

2 Cassandro, G., «Resistenza (diritto di)», in Noviss. Dig. it., vol. XV, Torino, 1968, p. 592. 
moniaco del potere, che, in quanto potere, in quanto forza, non può soffrire limite alcuno. È diffusa l'idea che il potere tanto più è potere quanto meno è limitato; e il potere assoluto è quello del tutto illimitato.

Ora potrebbe apparire onorifico (o, secondo i punti di vista, negativo) per la Chiesa, per il messaggio cristiano, essere identificati senza residui con l'ethos, essere trattati come la sede della giustizia e del diritto, in altri termini, come il limite al potere; potrebbe a qualcuno sembrare anche apprezzabile che taluni essenziali diritti (per non andare lontani, il diritto alla vita) siano soltanto difesi, in tutte le loro implicazioni e conseguenzialità, in un orizzonte religioso.

Ancora: potrebbe essere persino comodo fuggire dalle proprie responsabilità (che è come dire dalla propria umanità), ed affidare ad una chiesa, ad un movimento religioso, la tutela di interessi in realtà non delegabili, il limite al potere. E sarebbe più comodo per la forza, per il potere, rigettando l'influsso di una chiesa, di una religione, liberarsi d'un colpo dai limiti che con poca avvedutezza a tale influsso erano stati ricondotti e ridotti. E si potrebbe al riguardo anche osservare come la clericalizzazione del messaggio cristiano abbia avuto quale aberrante effetto riflesso la liberazione del demoniaco del potere.

Ma la verità è che la laicizzazione del diritto quasi nulla in realtà significa se è ridotta all'autonomia del potere civile dal religioso, $o$ alla aconfessionalità dello Stato, per certi aspetti doverosa e giustificabile. La laicità acquista il suo più vero e profondo significato solo all'interno del più ampio e universale problema della laicizzazione della forza e del potere.

È' stato ed è, più che un grave errore politico, una distorsione culturale, considerare l'etica come categoria confessionale, legata ad uno specifico profilo della condizione umana, quando essa è viceversa categoria universale, attinente all'uomo in quanto uomo, e non soltanto in quanto credente.

E certo la forza, il potere, svelati nel loro volto demoniaco, o organizzati in forme ipocrite che vorrebbero occultarli, si presentano anch'essi come profili essenziali, universali, della realtà umana.

A Giocasta, che esortava Eteocle, suo figlio, a non nutrire nel suo cuore l'Ambizione, la più trista delle divinità, e lo spronava ad avere viceversa in pregio l'Uguaglianza e ad onorarla, poiché solo ciò che è uguale può fare legge tra gli uomini, e lo implorava a non stimare la potenza di un regno, che altro non è - come ella diceva - se non un'ingiustizia congiunta con la ricchezza, Euripide (siamo nel V secolo a. C.) rispose mettendo in bocca ad Eteocle quelle parole che sono l'epigrafe della forza, la giustificazione e la glorificazione di 
ogni malintesa ragion di Stato: «Se c'è da compiere un'ingiustizia, ebbene la più bella delle ingiustizie è quella che dà il regno [i.e. il potere] $\gg^{3}$.

Il più alto egoismo (il fratello che non vuole condividere il regno con l'altro fratello, cui pure spettava), e quindi la più alta iniquità, a fondamento del potere; la forza, svincolata da ogni limite etico; ecco il demoniaco del potere.

I tentativi di razionalizzazione della forza, attraverso la progressiva emancipazione (riscontrabile in tutte le correnti dell'ellenismo) della moralità e della religione dalla polis, l'invocazione della sophrosune, l'equilibrata assennatezza, da opporre all'ossessione della forza (spesso rinvenibile nelle correnti stoiche del pensiero antico, in particolare in Cicerone), che per secoli e secoli costituirono il motivo costante intorno a cui si arrovellarono generazioni di pensatori, desiderosi di rappresentare il potere in forme giustificabili, tali da renderlo accettabile, sfociarono alla fine, col pensiero giudaico cristiano, nella proclamazione di un messaggio del tutto rivoluzionario: la soggezione dei potenti e dei loro atti, e cioè del potere e del suo concreto esercizio, al giudizio divino: «Porgete l'orecchio, voi che dominate le moltitudini e siete orgogliosi per il gran numero dei vostri popoli. La vostra sovranità proviene dal Signore; la vostra potenza dall'Altissimo, il quale esaminerà le vostre opere e scruterà i vostri propositi; poiché, pur essendo ministri del suo regno, non avete governato rettamente, né avete osservato la legge né vi siete comportati secondo il volere di Dio. Con terrore e rapidamente egli si ergerà contro di voi, poiché si compie un giudizio severo contro coloro che stanno in alto. L'inferiore è meritevole di pietà, ma i potenti saranno esaminati con rigore» $(\operatorname{Sap} 6,2-6)$.

Nasce in quel momento il bipolarismo nel pensiero politico occidentale: da una parte vi sono i potenti, vi è il potere, vi è la forza; ma, dall'altra parte, con non minore consistenza oggettiva, vi è la Legge, vi è la Giustizia, vi è (il disegno di) Dio; e l'uomo è per così dire liberato nello spirito dalla tirannia di un mondo cosmico monistico, ove alla forza poteva essere contrapposta soltanto l'esortazione ai potenti di un suo moderato esercizio, ma nessun limite oggettivo, nessun polo contrario.

Sant'Agostino, come nessun altro prima, fungendo da cerniera fra il pensiero del mondo antico, che andava illanguidendo, e del nuovo, vivificato e corroborato dal messaggio cristiano, fissò il problema in termini dai quali nei secoli futuri non ci si sarebbe più allontanati, se non per ricadere nella sfrena-

3 EurIPIDE, Le Fenicie, trad. di C. Diano, in Il teatro greco. Tutte le tragedie, Firenze, 1970, p. 883. 
tezza del potere. «Se la repubblica è res populi - e lo affermava Cicerone ${ }^{4}-$, e cioè cosa del popolo; e non vi è popolo se le persone non sono aggregate da un comune senso del diritto - anche questa era persuasione di Cicerone ${ }^{5}$-; ebbene, non v'è diritto senza la giustizia («non est ius, ubi nulla iustitia est»), e perciò, senza alcun dubbio, dove manca la giustizia manca la stessa repubblica («ubi iustitia non est, non esse rempublicam»)», proprio in quanto non si ha vero popolo ${ }^{6}$.

Qui non abbiamo più un'esortazione ai potenti, ma un limite razionale e morale al potere. La forza non può, non nel senso che non deve, ma nel senso che non riesce a fare la repubblica senza la giustizia, senza il rispetto dell'etica; solo la giustizia, il diritto, la morale, possono trasformare un'accozzaglia di individui in popolo; la forza, il potere, non possono, anche perché in essi diritto e giustizia non hanno il loro fondamento e la loro certa dimora. La giustizia e il diritto sono, infatti, categorie ideali autonome, da non confondere con la giustizia e il diritto espressioni del potere, siccome noi oggi siamo abituati a concepirli.

Quindici secoli prima di Santi Romano e del suo Ordinamento giuridico, sant'Agostino non avrebbe avuto difficoltà a riconoscere che anche le bande dei briganti (il Romano parlerà di società rivoluzionarie e di associazioni a delinquere $^{7}$ ) si presentavano come piccoli regni, schiere armate di uomini comandate da un capo, legate da un patto sociale, operanti secondo una legge da tutti accolta ${ }^{8}$; ma rimanevano, per l'Ipponate, bande di briganti, e non già perché una forza superiore (lo Stato, per Santi Romano; Alessandro il grande, nel celebre colloquio con il pirata ${ }^{9}$ ) tali le qualificava e come tali le trattava,

4 De re publica, 1.25.39, 32.48 (ed. Patavii, 1968, p. 20-21, 25-26).

$5 \mathrm{Ibid}$. Secondo la celebre definizione ciceroniana, il popolo infatti non è «omnis hominum coetus quoquo modo congregatus, sed coetus multitudinis iuris consensu et utilitatis communione sociatus» (1.25.39). E altrove Cicerone si chiederà: «Quid est enim civitas nisi iuris societas?» (1.32.49).

6 De Civ. Dei, 19, 21 (P.L. 41, 648-649). Per sant'Agostino, il diritto è legato non alla dimensione del potere, ma a quella della giustizia, secondo una persuasione diffusa nella tradizione neotestamentaria e in tutto il pensiero di ispirazione cattolica: «ubi (...) justitia vera non est, nec jus potest esse». Ciò, infatti, che è conforme al diritto - si noti la forza argomentativa del grande padre della Chiesa - è certo giusto; ma ciò che è contrario alla giustizia non può essere secondo il diritto («quod enim jure fit, profecto juste fit: quod autem fit injuste, nec jure fieri potest») (ivi).

7 Cfr., di tale A., L'ordinamento giuridico (1918), II ed. riv., Firenze, s.d. (1945), p. 36, 160.

8 De Civ. Dei, 4, 4 (P.L. 41, 115).

9 Il colloquio, accennato nel De re publica di Cicerone (3.14.24, ed. Patavii, cit., p. 77-78), fu ripreso da sant'Agostino nel De Civ. Dei (4, 4, cit.). Ad Alessandro Magno, da cui era stato fatto prigioniero, che gli chiedeva per quale ragione infestasse i mari, il pirata seppe rispondere: «Per la stessa ragione per cui tu infesti il mondo. Solo che io, con la mia misera nave, sono chiamato ladro, mentre tu, con la tua grande flotta, imperatore». 
ma proprio perché agivano senza rispettare la Giustizia. Grande o piccolo che fosse il regno, il gruppo organizzato, quale che fosse la sua potenza, senza il rispetto della giustizia, esso era un brigantaggio.

Questa alta concezione ideale, che per un millennio ispirò la vita politica europea, suggerendo ed esigendo un equilibrato assetto del rapporto ethos-kratos, quale mai più la società occidentale avrebbe conosciuto, perdette strada facendo - com'è noto - la sua forza rigeneratrice, in primo luogo nei fatti politici, attraverso una progressiva elusione delle vie della moralità, pur ammesse in principio, con il conseguente affrancamento del potere dai limiti esterni - che erano insieme limiti ideali e spirituali - della Giustizia e del Diritto.

Spettò a Machiavelli il merito di svelare ciò che era rimasto occulto, ma non inoperoso: il demone della forza. Ma la presa di coscienza del problema non servì - ed anche questo è noto - ad un risveglio non dico della coscienza morale, ma della ragione, per ritrovare il limite al potere.

Altri e noti eventi del pensiero avvolsero la ragione nel primo Rinascimento, ed essa non ebbe quasi il tempo di avvedersi della mortale minaccia che incombeva sul mondo dell'eticità. Da Bodin a Botero, da Boccalini a Tommaso Campanella a Gabriel Naudé, il machiavellismo, questa liberazione della forza dalla gabbia ove per secoli era stata ristretta entro i robusti ferri del mondo dell'eticità, ebbe avversari, anche agguerriti, ma nessuno capace di frenare la caduta della bipolarità cristiana ${ }^{10}$.

Alla fine, a conclusione di un travagliato e drammatico percorso culturale, l'uomo non sente più di far parte di una dimensione etica e giuridica dall'alto della quale giudicare il potere. La forza, il potere, in breve: lo Stato moderno, ormai lo sovrastano. La sua stessa coscienza morale ha origine dai precetti dello Stato. Il Diritto, la Giustizia (categorie ideali, con le iniziali maiuscole) sono alla fine diventati un diritto e una giustizia (con le iniziali minuscole), uno dei tanti diritti e delle tante giustizie che, come strumenti operativi, derivano dalla giurisdizione di chi ha in mano la forza, e dipendono dall'arbitrio che la governa.

Queste le nuove consapevolezze. Ecco, in ultimo, il diritto laico. Ł̀ questo il diritto del potere, un potere affrancato da ogni limite oggettivo; è, precisamente, il diritto fondato sul potere; è il diritto assoggettato soltanto al potere; è, infine, il diritto che pretende di dare origine al nostro stesso mondo morale.

10 Sugli autori nominati, e su Machiavelli in primo luogo, cfr. MEINECKE, F., L'idea della ragion di stato nella storia moderna, trad. it. di D. Scolari, Firenze, 1970, pp. 25-205. 
$\grave{\mathrm{E}}$, in breve, il diritto moderno, che laico è per essenza, qualunque ne sia il contenuto.

In questa visione complessiva trova spiegazione l'enunciata contraddittoria e, per certi versi, misteriosa ambivalenza della laicità. Un'ambivalenza che risulta evidente (non si spiega), quando si voglia definire la laicità dai contenuti del diritto, ch'essa connoterebbe, ma che svanisce, quando si pensi alla sua natura o essenza.

Laico, senza contraddizione alcuna, sarà il diritto che fa aggio sulla forza, qualunque cosa, anche contrastante, esso disponga: laico sarà pertanto il diritto ispirato alla aconfessionalità dello Stato o anche espressione di una politica antiecclesiastica; ma parimenti laico sarà il diritto favorevole al fenomeno religioso, se, nell'uno e nell'altro caso, è concepito come espressione di un potere per se stesso etico. Può non esservi contraddizione alcuna tra l'idea della laicità, espressa dalla Corte Costituzionale italiana - con la sua affermazione di uno Stato cui non può essere inibito, in base al diritto della Carta fondamentale, di prestare un servizio alla dimensione religiosa -, e, poniamo, le idee fieramente avverse alla Chiesa cattolica, manifestate da un Guido Padelletti o da un Francesco Scaduto ${ }^{11}$. L'ambiguità è soltanto apparente. Il diritto, nel mondo ideale rappresentato da entrambe le concezioni, non è fonte di obblighi giuridici ed etici, perché valide ed accettabili sono le sue prescrizioni sulla base di criteri assoluti, trascendenti il diritto stesso, ma perché ha la forza di imporli, perché esso altro non è se non «l'utile del potere costituito», come affermava Trasimaco nella Repubblica di Platone ${ }^{12}$.

Dire che questa potenza, questa forza, sono cresciute a dismisura, e per questo vanno paventate, è come dir nulla o fare un inutile rilievo moralistico, se non si comprende che la crescita della forza non si misura dalla sua capacità distruttiva, dalla sua potenza di incidere nel regno della umana natura, ma proprio dal venire meno dei limiti ad essa posti.

Le masse umane, assoggettate alla forza, sono state nell'epoca a noi contemporanea, in larghe parti del mondo, assimilate alla forza, ad accre-

11 Di tali A. si leggano le rispettive prolusioni romane: Padelletti, G., «Roma nella storia del diritto», in Arch. giur., 12 (1874), pp. 191-223; SCADUTO, F., Stato e confessioni religiose, Roma, 1912.

12 I, 339a (trad. di F. Sartori, in Platone, Opere complete, vol. VI, Bari, 1971, p. 51). Un potere che non si fonda nella ragione, ma sulla forza: $\ll \mathrm{Ma}$, se non erro, questo potere detiene la forza: così ne viene, per chi sappia bene ragionare - precisava Trasimaco -, che in ogni cosa il giusto è sempre l'identica cosa, l'utile del più forte». 
scerne la potenza e l'indiscutibilità, attraverso meccanismi di giustificazione del potere (la volontà generale del popolo, che fonda le leggi e persino le sentenze) mistificanti ma efficaci. Chi oggi si sentirebbe di sostenere che non vi è popolo senza la Giustizia, ma «qualiscumque multitudo, quae populi nomine digna non est ${ }^{13}$ ? Chi oggi penserebbe ad una indipendenza, se non ad una precedenza ideale della Giustizia e del Diritto (tornati con le iniziali maiuscole) rispetto al popolo? Ed oggi il «popolo» (con la «pubblica opinione» che ne vuole rappresentare la volontà, quasi fosse un essere antropomorfizzato), anche se inteso come concetto sociologico, sotto il profilo etico del tutto neutro, è pur sempre considerato, da estesi settori della cultura contemporanea, come la fonte convenzionale suprema della stessa eticità.

$\mathrm{Ma}$ - per andare alla radice ultima del positivismo imperante - quanti, fuori di una ispirazione di fede, riescono ad affermare sul piano filosofico (come seppero affermarli la cultura greca classica nei picchi più alti della sua riflessione metafisica, e la philosophia perennis di matrice cristiana) qualcosa o Qualcuno che antecede e trascende l'uomo e la sua volontà particolare, il popolo e la sua volontà generale, lo Stato con il suo spirito oggettivo e la legge da esso posta, in breve: il mondo dell'immanenza, dal pensiero moderno concepito come del tutto autosufficiente, l'unico mondo razionale?

Non sono certo mancati, dal momento in cui il bipolarismo cristiano si è oscurato nello spirito umano, e questo di esso più non vive, $i$ tentativi volti a razionalizzare il potere, al fine di limitarne la forza, di imbrigliarne se non di sconfiggerne gli aspetti demoniaci. Dal giusnaturalisno razionalista, di matrice protestante, al pensiero illuministico, dal sociologismo razionale francese (Duguit), al sociologismo pragmatico americano, si è voluto in qualche modo porre un limite alla forza. «Invano», gridano sterminate masse di schiavi e di morti dei nostri tempi.

Ma perché invano?

Grandi storici hanno ritenuto che a guidare la forza, che ormai costituisce l'anima stessa e non solo il braccio della ragion di Stato, sia bene udire, sempre e con serietà ed attenzione, la lingua - com'essi dicono - del puro idealismo cristiano, «con il dolore - aggiungono - che il mondo non si possa cambiare per mezzo di essa ${ }^{14}$.

13 De Civ. Dei, 19, 21, cit.

14 MeINECKE, F., Lidea della ragion di stato nella storia moderna, cit., p. 435. 
Altri, in una visione più ristretta, più meccanica, delle forze ideali che agiscono nel mondo, una visione che ormai non dovrebbe esserci difficile ricondurre ai problemi, anzi al problema di fondo del pensiero politico - giuridico occidentale, notano come la società moderna, privata d'ogni direzione morale, non sappia più quali leggi possano essere atte a dirigere l'umanità ${ }^{15}, \mathrm{e}$ per implicito auspicano che si torni ad avere un diritto che ammetta la forza creatrice di una morale trascendente ${ }^{16}$; morale che, peraltro, in ossequio alle dominanti categorie dogmatiche, potrebbe essere ammessa soltanto se trasfusa nel costume del popolo.

Sono come richiami rituali, volti a tranquillizzare le nostre cattive coscienze, e, per il resto, vaniloqui. Il vero è che essi ruotano tutti intorno ad un'idea del potere e della forza senza limiti; ci si illudeva che la legittimazione del potere, secondo le categorie politico - giuridiche che si affermano nel corso della storia, possa bastare a giustificare il suo operato e le sue manifestazioni. Ma sono appunto illusioni. Sfugge a quelle proposizioni l'essenziale, vale a dire che il demoniaco del potere non inerisce a questo o a quel regime politico, a questa o a quella forma dello $\operatorname{Stato}^{17}$, ma alla persuasione che nella forza, nello Stato, è incarnata la ragione morale, anzi, come dirà Hegel senza infingimenti, che «lo Stato è la stessa realtà dell'idea etica» ${ }^{18}$, e che la realtà etica, che esiste come Stato, alla fine trapassa «come contenuto (...) nella forma dell'universalità», cioè nella legge ${ }^{19}$; onde, come è stato osservato, la legge dello Stato «è, per la coscienza di chi rifletta, verità morale» ${ }^{20}$.

Non si smontano coteste convinzioni se diritto e giustizia non tornano a rappresentare categorie spirituali di un mondo ideale indipendente dal potere, se continuano ad essere concepiti come meri strumenti tecnici che riflettono, secondo il pensiero del filosofo di Stoccarda, la verità morale che è nella forza.

15 RIPERT, G., Les forces créatrices du droit, Paris, 1955, p. 157.

16 Ibid., p. 188; infatti, secondo l'A., un diritto che non vuole ammettere la forza creatrice di una morale trascendente deve rassegnarsi a non chiedere il soccorso della regola morale per assicurare la sua osservanza.

17 Per un'osservazione di tal genere, cfr. RitTer, G., Die Dämonie der Macht, München, 1948, trad. it. di E. Melandri, Il volto demoniaco del potere, III ed., Bologna, 1971, p. 139.

18 Hegel, G. W. F., Grundlinien der Philosophie des Rechts (1821), \$257, trad. it. di G. Marini, Lineamenti di filosofia del diritto, Bari, 1987, p. 195.

19 Ibid., $\$ 270$ (trad. cit., p. 195).

20 RitTer, G., Die Dämonie der Macht, trad. cit., p. 147. 
In tale ottica poteva Benedetto Croce chiedersi, all'indomani di quella spaventosa tragedia dell'umanità che fu la prima guerra mondiale: «Perché mai si è fatta la guerra, se non per vivere in guisa più piena, più degna, più alta, più possente? Tutti, vincitori e vinti - continuava egli - respiriamo certamente una vita spirituale superiore a quella di prima della guerra $»^{21}$. E questo pensiero induceva un vinto, un grande vinto, ad esclamare: «Noi vinti, sentiamo nei fatti, ma con intima commozione, il giusto di questa constatazione» ${ }^{22}$. A un secolo di distanza confesso di non riuscire a vedere il giusto nell'affermazione crociana. Avverto anzi la sua profonda ingiustizia; e il fatto d'essere essa distante dai sentimenti umanitari del suo autore, la esalta come espressione di una crudeltà quasi metafisica, inesorabile, nei confronti della quale nulla può l'uomo. Questa forza, che manifestò la sua più cupa ferocia nella guerra, e che, nonostante tutto, era apprezzata come portatrice di verità morale (in ciò Croce era compiutamente hegeliano), tanto da indurre i superstiti dal massacro a respirare «una vita spirituale superiore», è stata non solo la rovina materiale, ma anche la rovina spirituale del mondo.

Ma si badi non la forza, non il potere in sé, ma l'averli ritenuti custodi della verità morale; ma le idee che hanno portato il diritto, la giustizia ad appiattirsi in essi, a divenire «laici», nell'unico significato non convenzionale conosciuto, a togliere loro la funzione di limite oggettivo del kratos; una funzione che consentiva all'uomo (e potrebbe ancora consentirgli) di concepire la nozione e la forza della libertà come verità morale, quella libertà, non passionale ma razionale, che consiste nella possibilità di giudicare il potere, poiché nel giudizio e nella sua possibilità, come che questo si concluda, anche con la perdita della testa (è il caso di Antigone, è il caso di Tommaso Moro), è il limite radicale del potere.

21 Croce, B., Scritti varii, vol. III: L'Italia dal 1914 al 1918. Pagine sulla guerra, IV ed., Bari, 1965, p. 295. Sarebbe errato da ciò dedurre che Croce fosse un partigiano della guerra; egli, al contrario, si oppose all'intervento italiano (almeno fin quando questo non avvenne) e rispose con grande vigore morale e politico agli argomenti di quanti in quell'intervento vedevano una necessità, l'unico modo per affermare l'esistenza dell'Italia come nazione, considerato che la sua unità si era realizzata nel Risorgimento più per fortunate circostanze, che non per valore. Così ragionava il Croce cittadino; ma Croce filosofo - alla distinzione egli teneva: vedi p. 41-42 della citata opera -, il Croce che voleva rendersi conto e dar conto, sotto un profilo speculativo e teorico, di quel che stava avvenendo nella vita dello spirito, credeva - non per auspicare, ma per spiegare - che, con lo spargimento del «miglior sangue» europeo, potesse «maturare più alta forma di civiltà» (vedi il Manifesto da lui scritto, affisso a Napoli, con data 3 maggio 1915: op. cit., pp. 48-50).

22 MeINECKE, F., L'idea della ragion di stato nella storia moderna, cit., p. 443. 
Temo che il limite della forza (nel senso di limite alla forza) non sia più avvertito come problema ideale e culturale. Il diritto è trionfalmente laico, poiché la forza è senza limiti. Si pensa che alla forza possa essere limite soltanto un'altra forza. E alla stessa Chiesa si guarda non più per i valori che incarna, e che, in quanto valori universali, vivono anche fuori di essa, ma per la forza, per il potere che può dispiegare, se può dispiegarlo.

Si è perduta così l'idea di un Diritto, di una Giustizia, come limiti spirituali, oggettivi, al potere, non da esso discendenti. Pure, se una qualche luce dovrà venire a rischiarare il cielo del terzo millennio appena iniziato, occorrerà riuscire a pensare il diritto non come il diritto della forza, nella forza assiso, la giustizia non come giustizia voluta dal potere, dal potere derivante. 